Surveys in Differential Geometry XIV

\title{
Geometry of Teichmüller space with the Teichmüller metric
}

\author{
Howard Masur
}

\section{Introduction}

The purpose of this chapter is to describe recent progress in the study of Teichmüller geometry. We focus entirely on the Teichmüller metric. A survey of the very important Weil-Petersson metric can be found in $[\mathbf{W}]$. The study of the Teichmüller metric has different aspects. One major theme in the subject is to what extent Teichmüller space with the Teichmüller metric resembles a metric of negative curvature, and to what extent it resembles a metric of nonnegative curvature. This theme will occupy much of this survey and we will describe results in both directions. With somewhat the same theme we describe recent results about the Teichmüller geodesic flow on moduli space.

Along somewhat different lines we describe some recent important work of K. Rafi that gives a combinatorial description of the Teichmüller metric. Another important subject is the study of the action of the action of the mapping class group on Teichmüller space. We will discuss some very important recent work of Eskin, Mirzakhani and co-authors on counting problems for the mapping class group.We will also describe some recent joint work with Benson Farb on the Teichmüller geometry of moduli space.

There has also been a great deal of recent work on the related topics of the $\operatorname{SL}(2, \mathbf{R})$ action on spaces of quadratic differentials, Veech groups and Veech surfaces. These fall outside the scope of this article. We refer to the article of Hubert, Lanneau and Moeller in these proceedings for a discussion of these last subjects.

For general references for Teichmüller theory, and quasi-conformal mappings I refer to the books of L. Ahlfors $([\mathbf{A}])$, J. Hubbard $([\mathbf{H}])$ and A. Papdoupolous $([\mathbf{P}])$. For a reference to the mapping class group I refer to the book of N. Ivanov ([I1] and the recent book of B. Farb and D. Margalit $([\mathbf{F M a}])$. For references to the theory of quadratic differentials there are

Author is supported in part by the NSF.

(C)2009 International Press 
the books of Strebel $([\mathbf{S t}])$ and F. Gardiner $([\mathbf{G}])$. See the paper of L. Bers ([B1]) for a discussion and proof of Teichmüller's theorem and $[\mathbf{F L P}]$ for a discussion of measured foliations and Thurston's boundary of the mapping class group.

I would also like to thank the referee for several helpful comments.

\section{Preliminaries}

Let $S=S_{g, n}$ be a surface of genus $g$ with $n$ punctures. To avoid sporadic cases we will assume $3 g-3+n>0$. In some cases we will assume $3 g-3+n>$ 1 so that we avoid the cases of the once punctured torus and four times punctured sphere. A complex structure or Riemann surface structure $X$ on $S$ is an atlas of charts

$$
\left\{z_{\alpha}: U_{\alpha} \rightarrow \mathbf{C}\right\}
$$

such that the transition functions $z_{\beta}^{-1} \circ z_{\alpha}$ are biholomorphic, where defined. Then the Teichmüller space of $S$ denoted Teich $(S)$ is the space of complex structures $X$ on $S$ up to equivalence. We say that $X \sim Y$ if there is a map $f: X \rightarrow Y$, biholomorphic in the coordinate charts, which is isotopic to the identity on $S$.

By the uniformization theorem, each point $X$ in $\operatorname{Teich}(S)$ has a metric of constant curvature -1 , and so equivalently, we can describe Teich $(S)$ as the space of hyperbolic metrics $\rho$ of constant curvature -1 on $S$ up to equivalence, where $\rho_{1} \sim \rho_{2}$ if there is an isometry of $S$ isotopic to the identity taking $\rho_{1}$ to $\rho_{2}$. A theme of much of Teichmüller theory is to compare the complex analytic theory where points are given by Riemann surfaces and the hyperbolic geometry. Since the correspondence is given by the uniformization theorem, there are rarely exact formulae and one often has to rely on estimates in making comparisons.

Given a hyperbolic structure $\rho$ on $S$ there is a unique geodesic in the homotopy class of every simple closed curve $\alpha$. We denote by $\ell_{\rho}(\alpha)$ the length of the geodesic. For $X$ a Riemann surface we will also write $\ell_{X}(\alpha)$, where by this we mean we have given $X$ its hyperbolic structure. We will let $\mathcal{S}$ be the set of homotopy classes of homotopically nontrivial essential simple closed curves on $S$.

The Teichmüller metric on Teich $(S)$ is the metric defined by

$$
d_{T}(X, Y):=\frac{1}{2} \inf _{f \sim I d}\{\log K(f): f: X \rightarrow Y\}
$$

where $f$ is quasiconformal and

$$
K(f):=\left\|K_{x}(f)\right\|_{\infty} \geq 1
$$

is the quasiconformal dilatation of $f$, where

$$
K_{x}(f):=\frac{\left|f_{z}(x)\right|+\left|f_{\bar{z}}(x)\right|}{\left|f_{z}(x)\right|-\left|f_{\bar{z}}(x)\right|}
$$

is the pointwise quasiconformal dilatation at $x$. 
GEOMETRY OF TEICHMÜLLER SPACE WITH THE TEICHMÜLLER METRIC 297

The mapping class group $\operatorname{Mod}(S)$ is the group of homotopy classes of orientation-preserving homeomorphisms of $S$. This group acts properly discontinuously and isometrically on $\operatorname{Teich}(S)$ with the Teichmüller metric and so the quotient

$$
\mathcal{M}(S)=\operatorname{Teich}(S) / \operatorname{Mod}(S)
$$

has the induced metric. The space $\mathcal{M}(S)$ is the moduli space of (unmarked) Riemann surfaces, or what is the same thing, conformal structures on $S$.

Given a Riemann surface $X$ and $\alpha \in \mathcal{S}$, the extremal length of $\alpha$ on $X$ is defined by

$$
\operatorname{Ext}_{X}(\alpha)=\sup _{\sigma} \frac{L_{\sigma}^{2}(\alpha)}{A(\sigma)}
$$

where the supremum is over conformal metrics $\sigma(z)|d z|$,

$$
L_{\sigma}(\alpha)=\inf _{\alpha^{\prime} \sim \alpha} \int_{\alpha^{\prime}} \sigma(z)|d z|
$$

and

$$
A(\sigma)=\int_{X} \sigma^{2}(z)|d z|^{2}
$$

The following formula of Kerckhoff ([Ke]) has proven to be extremely useful in estimating Teichmüller distances.

$$
d_{T}(X, Y)=\sup _{\alpha \in \mathcal{S}} \frac{1}{2} \log \frac{\operatorname{Ext}_{X}(\alpha)}{\operatorname{Ext}_{Y}(\alpha)} .
$$

2.1. Quadratic differentials and Teichmüller rays. Let $X$ be a Riemann surface. A (meromorphic) quadratic differential $q$ on $X$ is a tensor locally given by $q=q(\zeta) d \zeta^{2}$, where $q(\zeta)$ is a meromorphic function of the holomorphic coordinate $\zeta$. A quadratic differential has a finite number of zeroes and poles. We allow simple poles at the punctures of $S$. The number of zeroes and poles of $q$ counting multiplicity is $4 g-4$. A simple pole has multiplicity -1 . In a neighborhood of a regular point $p$ choose a coordinate $\zeta$ with $p$ corresponding to $\zeta=0$ and take a branch of $q^{1 / 2}(\zeta)$. Define

$$
z(\zeta)=\int_{0}^{\zeta} q^{1 / 2}(u) d u
$$

Then $z$ becomes a new natural coordinate in a neighborhood of $p$ and in this coordinate, $q$ is given by $q(\zeta) d \zeta^{2}=d z^{2}$. In a neighborhood of a zero of order $k \geq 1$ there are natural coordinates such that $q(\zeta) d \zeta^{2}=z^{k} d z^{2}$. The set of quadratic differentials on $X$ is denoted by $\mathrm{QD}(X)$ and is a complex vector space of dimension $3 g-3+n$.

The fact that there are natural coordinates $z$ so that $q(\zeta) d \zeta^{2}=d z^{2}$ allows one to say that $q$ defines a local Euclidean metric $|d z|^{2}$, with finitely many singular points corresponding to the zeroes and poles of $q$. At a zero of order $k$, the metric is not Euclidean; there is a cone angle singularity. The cone angle of the metric is $\pi(k+2)$. The total area of $X$ in this metric is finite. The 
area of the surface induced by the quadratic differential is denoted by $\|q\|$. This defines a norm on $\mathrm{QD}(X)$. A saddle connection is a geodesic segment joining two (not necessarily distinct) singular points which has no singular points in its interior.

The description of a quadratic differential in terms of a Euclidean metric also allows one to describe quadratic differentials in purely geometric terms as consisting of a collection of polygons $\Delta_{1}, \ldots, \Delta_{N}$ in the plane with the property that for every side $s_{i}$ of $\Delta_{i}$ there is a unique side $s_{j}$ of some $\Delta_{j}$ such that $s_{i}$ is glued to $s_{j}$ by a translation or a rotation by $\pi$ followed by a translation. The polygons then provide the natural coordinates $z$ and the fact that the side gluings are of the given form means that the overlap functions are of the form

$$
z \rightarrow \pm z+c
$$

so that $q=d z^{2}$ is really a quadratic differential. The vertices of the polygons give rise to zeroes and simple poles of the quadratic differential. If there are no rotations, then the resulting object is called a translation surface and corresponds to an Abelian differential $\omega$ on a Riemann surface $X$ with $\omega^{2}=q$ a quadratic differential.

Consider the data $\hat{k}=\left(k_{1}, \ldots, k_{n}, \pm\right)$ where the $k_{i}$ are the orders of the zeroes and poles and one has a $+\operatorname{sign}$ if $q$ is the square of an Abelian differential and - sign if not. The set of all quadratic differentials over Teich $(S)$ with data $\hat{k}$ is called a stratum and is denoted $\mathrm{QD}_{\hat{k}}$. As $\hat{k}$ varies, these strata fit together to form the bundle QD of all quadratic differentials over $\operatorname{Teich}(S)$. We denote by $\mathrm{QD}_{\hat{k}}^{1}$ the set of elements $q \in \mathrm{QD}_{\hat{k}}$ with $\|q\|=1$. In the compact case the principal stratum corresponds to $\hat{k}=(1, \ldots, 1,-)$. The mapping class group acts on each stratum $\mathrm{QD}_{\hat{k}}$ with quotient denoted $\mathbf{Q}_{\hat{k}}$.

A quadratic differential also determines a pair of transverse measured foliations $F_{h}(q)$ and $F_{v}(q)$, called the horizontal and vertical foliations for $q$. In the natural coordinates $z=x+i y$, away from the zeroes, the leaves of $F_{h}(q)$ are given by

$$
y=\text { const; }
$$

and the leaves of $F_{v}(q)$ are given by

$$
x=\text { const }
$$

and the transverse measures are $|d y|$ and $|d x|$. The foliations $F_{h}(q)$ and $F_{v}(q)$ have the zero set of $q$ as their common singular set, and at each zero of order $k$ they have a $(k+2)$-pronged singularity, locally modelled on the singularity at the origin of $z^{k} d z^{2}$. The leaves passing through a singularity are the singular leaves of the measured foliation.

Theorem 2.1 (Teichmüller's Theorem). Given any $X, Y \in \operatorname{Teich}(S)$, there exists a unique quasi-conformal map $f$, called the Teichmüller map, such that

$$
d_{T}(X, Y)=\frac{1}{2} \log K(f) .
$$


Furthermore the Beltrami coefficient $\mu:=\frac{f_{\bar{z}}}{f_{z}}$ is of the form $\mu=k \frac{\bar{q}}{|q|}$ for some unique $q \in \mathrm{QD}^{1}(X)$ and some $k$ with $0 \leq k<1$. The quadratic differential $q$ is called the initial quadratic differential of the map. There is a quadratic differential $q^{\prime} \in \mathrm{QD}^{1}(Y)$ called the terminal quadratic differential with the following properties. The map $f$ takes zeroes of $q$ to zeroes of $q^{\prime}$ of the same order. In the natural local coordinates $z=x+i y$ of $q$ away from the zeroes, and the natural coordinates $w=u+i v$ for $q^{\prime}$,

$$
u=K^{1 / 2} x, v=K^{-1 / 2} y,
$$

where $K=K(f)=\frac{1+k}{1-k}$.

Conversely, given a Riemann surface $X$, a quadratic differential $q \in$ $\mathrm{QD}^{1}(X)$ and $t \geq 0$, there is a Riemann surface $X_{t}$ and a terminal quadratic differential $q_{t}$ on $X_{t}$ for a Teichmüller map $f_{t}: X \rightarrow X_{t}$ such that

$$
d_{T}\left(X, X_{t}\right)=t
$$

Define $K$ by $t=\frac{1}{2} \log K$. The Teichmüller map $f$ expands along the leaves of the horizontal foliation $F_{h}(q)$ by $K^{1 / 2}$ and contracts along the leaves of the vertical foliation $F_{v}(q)$ by $K^{-1 / 2}$. Set $q_{t}=q^{\prime}$. The family $X_{t}, t \geq 0$ is the Teichmüller geodesic ray $r(t)$ in Teich $(S)$ determined by $q$. The quadratic differentials $q_{t}$ all lie in the same stratum $\mathrm{QD}_{\hat{k}}$ and

$$
q \rightarrow g_{t}(q):=q_{t}
$$

is called the Teichmüller geodesic flow. It projects to a flow on $\mathbf{Q}_{\hat{k}}$ over moduli space.

The Teichmüller flow is part of a larger $\operatorname{SL}(2, \mathbf{R})$ action on each stratum. One can see the $\operatorname{SL}(2, \mathbf{R})$ action as follows. We realize $q$ as a union of glued polygons $\Delta_{1}, \ldots, \Delta_{p}$. A matrix $A \in \mathrm{SL}(2, \mathbf{R})$ acts on each polygon linearly, taking parallel sides of the same length to parallel sides of the same length. Then $A \cdot q$ is the union of the glued polyogns $A\left(\Delta_{1}\right), \ldots, A\left(\Delta_{p}\right)$. A great deal of recent work concerns this action. See the article of Hubert-LanneauMoeller in this volume.

We say a quadratic differential is Strebel if all of the leaves of the vertical foliation $F_{v}(q)$ are closed. In this case the surface decomposes into a finite number of Euclidean cylinders each swept out by freely homotopic closed vertical leaves of the same length. The boundary of the cylinders consist of a finite number of saddle connections and is called the critical graph. We say $q$ is mixed Strebel if the vertical foliation $F_{v}(q)$ contains at least one Euclidean cylinder. We will call a ray $r(t)$ (mixed) Strebel if it is determined by a (mixed) Strebel differential.

2.2. Measure on $\mathrm{QD}_{\hat{k}}$. Suppose $q_{0} \in \mathrm{QD}_{\hat{k}}$ where $\hat{k}=\left(k_{1}, \ldots, k_{p}, \pm\right)$ We represent $q_{0}$ as a union of glued polygons in some fashion. We may now find all $q \in \mathrm{QD}_{\hat{k}}$ in a neighborhood of $q_{0}$ by varying the polygons but keeping the corresponding side gluings. We choose an orientation of each side $s$ of 
each polygon. This gives a collection of vectors. A subcollection $\left\{s_{i}\right\}$ of these vectors determines each $q \in Q D_{\hat{k}}$ in a neighborhood of $q_{0}$. This gives a local embedding of $\mathrm{QD}_{\hat{k}}$ into $\mathbf{R}^{2 N}$ for an appropriate $N$. We pull back normalized

Lebesgue measure from $\mathbf{R}^{2 N}$ giving a measure $\mu_{\hat{k}}$ on $\mathrm{QD}_{\hat{k}}$. The measure is invariant under the action of $\operatorname{Mod}(S)$ giving a measure again denoted $\mu_{\hat{k}}$ on $\mathbf{Q}_{\hat{k}}$. Since $\mathrm{SL}(2, \mathbf{R})$ preserves Lebesgue measure, it is easily checked that the measure $\mu_{\hat{k}}$ is invariant under the $\operatorname{SL}(2, \mathbf{R})$ action on $\mathbf{Q}_{\hat{k}}$.

\section{Curvature aspects of Teichmüller geometry}

In this section we summarize much of what is known about the geometry of Teich $(S)$ with the Teichmuller metric. It follows from the uniqueness of the Teichmüller map that Teich $(S)$ is a straight space. This means that between any two points there is a unique Teichmüller geodesic which can be uniquely extended infinitely in both directions.

We begin with some old theorems. A geodesic metric space has Busemann negative curvature if for any two geodesic rays $r_{1}, r_{2}$ through a common basepoint $r_{1}(0)=r_{2}(0)$, and any $t>0$,

$$
d\left(r_{1}(t), r_{2}(t)\right) \leq \frac{1}{2} d\left(r_{1}(2 t), r_{2}(2 t)\right) .
$$

Theorem 3.1. ([M1]) For $3 g-3+n>1$, the Teichmüller metric does not have negative curvature in the sense of Busemann.

In fact it follows from work of Strebel $([\mathbf{S t}])$ that on any surface $X$ and collection of disjoint simple closed curves $\alpha_{1}, \ldots, \alpha_{p}$ there is a $p-1$ dimensional family of Strebel differentials in $\mathrm{QD}^{1}(X)$ determining cylinders in the homotopy classes of $\alpha_{1}, \ldots, \alpha_{p}$. For $p>1$ the corresponding rays $r_{1}, r_{2}$ satisfy $\sup d\left(r_{1}(t), r_{2}(t)\right)<\infty$.

A geodesic metric space is Gromov hyperbolic if there is $\delta$ such that for any geodesic triangle with vertices $x, y, z$ the side $[x y]$ is contained in the $\delta$ neighborhood of $[x z] \cup[y z]$.

TheOREM 3.2. ([MW]) Teich $(S)$ with the Teichmüller metric is not Gromov hyperbolic.

In fact the lack of hyperbolicity can be explained more fully by a result of Minsky's. ([Mi] ) Fix $\epsilon>0$ smaller than the Margulis constant for hyperbolic surfaces. This means that two curves with hyperbolic length smaller than $\epsilon$ must be disjoint.

Let $\mathcal{C}=\left\{\gamma_{1}, \ldots, \gamma_{p}\right\}$ be a collection of distinct, disjoint, nontrivial homotopy classes of simple closed curves. Let

$$
\Omega_{\mathcal{C}}(\epsilon):=\left\{X \in \operatorname{Teich}(S): \ell_{X}\left(\gamma_{i}\right)<\epsilon \text { for each } i=1, \ldots, p\right\} .
$$

Extend $\mathcal{C}$ to a maximal collection $\left\{\gamma_{1}, \ldots, \gamma_{d}\right\}$ of homotopy classes of disjoint simple closed curves. Let $\left.\left\{\theta_{i}, \ell_{i}\right)\right\}$ denote the corresponding 
GEOMETRY OF TEICHMÜLLER SPACE WITH THE TEICHMÜLLER METRIC 301

Fenchel-Nielsen coordinates on $\Omega_{\mathcal{C}}(\epsilon)$. The $\theta_{i}$ are certain twist coordinates. The Fenchel-Nielsen coordinates give global coordinates on Teich $(S)$; henceforth we will identify points in Teich $(S)$ with their corresponding coordinates.

Consider the Teichmüller space Teich $(S \backslash \mathcal{C})$, which is the space of complete, finite area hyperbolic metrics on $S \backslash \mathcal{C}$. Note that the coordinates $\left\{\left(\theta_{i}, \ell_{i}\right): i>p\right\}$ give Fenchel-Nielsen coordinates on $\operatorname{Teich}(S \backslash \mathcal{C})$.

Let

$$
\Phi=\left(\Phi_{1}, \Phi_{2}\right): \Omega_{\mathcal{C}}(\epsilon) \rightarrow \operatorname{Teich}(S \backslash \mathcal{C}) \times \prod_{i=1}^{p} \mathbf{H}^{2}
$$

be defined by

$$
\Phi\left(\left(\theta_{1}, \ldots, \theta_{d}, \ell_{1}, \ldots, \ell_{d},\right)\right):=\left(\theta_{p+1}, \ldots, \theta_{d}, \ell_{p+1}, \ldots, \ell_{d},\right) \times \prod_{i=1}^{p}\left(\theta_{i}, 1 / \ell_{i}\right) .
$$

We are changing the last set of length coordinates from $\ell$ to $1 / \ell$ giving coordinates in the upper half-space model of $\mathbf{H}^{2}$. We give $\mathbf{H}^{2}$ the metric $d s^{2}=\frac{1}{4}\left(d x^{2}+d y^{2}\right) / y^{2}$. The factor of $\frac{1}{4}$ leads to a factor of $\frac{1}{2}$ in the distance, and is consistent with the factor of $\frac{1}{2}$ in the Teichmüller metric. If $S \backslash \mathcal{C}$ is disconnected, then Teich $(S \backslash \mathcal{C})$ is itself a product of the Teichmüller spaces of the components of $S \backslash \mathcal{C}$; we endow this total product space itself with the sup metric, denoted by $d$. We remark that $\Phi$ is a homeomorphism onto its image, and its image is $\operatorname{Teich}(S \backslash \mathcal{C}) \times \prod_{i=1}^{p}\left\{\left(x_{i}, y_{i}\right) \in \mathbf{H}^{2}: y_{i}>1 / \epsilon\right\}$.

THEOREM 3.3. ([Mi]) With notation as above, there exists $D$ such that for all $X, Y \in \Omega_{\mathcal{C}}(\epsilon)$,

$$
\left|d(\Phi(X), \Phi(Y))-d_{T}(X, Y)\right| \leq D .
$$

This product structure with the sup metric allows one to map large balls $B_{R}$ in $\mathbf{R}^{k}$ for some $k>1$, with the Euclidean metric $d_{E}(\cdot, \cdot)$ quasiisometrically to Teich $(S)$. This means that there are constants $K, C$ and for all large $R$, a map $F: B_{R} \rightarrow \operatorname{Teich}(S)$ such that

$$
\frac{1}{K} d_{E}(x, y)-C \leq d_{T}(F(x), F(y)) \leq K d_{E}(x, y)+C .
$$

We now give evidence of negative curvature. For each homotopy class $\alpha$ of a simple closed curve, let Thin $(\alpha)$ denote the set of $X \in \operatorname{Teich}(S)$ such that $\ell_{X}(\alpha)<\epsilon$. We form the electrified Teichmüller $\operatorname{space}$ Teich $_{e l}(S)$ by adding a single point $x_{\alpha}$ for each homotopy class $\alpha$ and assigning distance 1 from $x_{\alpha}$ to each point of Thin $(\alpha)$.

Theorem 3.4. ([MM]) The electrified space $\operatorname{Teich}_{e l}(S)$ is a Gromov hyperbolic space. 
This Theorem is really a Corollary of work on the curve complex $\mathbf{C}(S)$. The vertices of the curve complex are isotopy classes of homotopically nontrivial, essential simple closed curves on $S$; namely the set $\mathcal{S}$. A $k$ simplex consists of $k+1$ mutually disjoint homotopically distinct simple closed curves. The dimension of $\mathbf{C}(S)$ is $3 g-4+n$. We are interested in the 1 skeleton $\mathbf{C}^{1}(S)$.

There is a map

$$
\mathbf{C}^{1}(S) \rightarrow \operatorname{Teich}_{e l}(S)
$$

which sends $\alpha$ to the point $x_{\alpha}$. The above theorem is a consequence of the theorem below.

TheOREM 3.5. ([MM]) The space $\mathbf{C}^{1}(S)$ is a Gromov hyperbolic space and the above map is a quasi-isometry.

Now the Teichmüller metric is not Riemannian but rather a Finsler metric which means that there is a norm on the tangent and cotangent spaces. The cotangent space at a point $X$ is the space $\mathrm{QD}(X)$ of quadratic differentials on $X$ and the norm of $q$ is $\|q\|$, the area of the flat metric defined by $q$.

We next describe some recent work on the geometry of sets in Teich $(S)$. Perhaps surprisingly, detailed information is sometines unknown. For example, it is not known if balls are convex. It is not even known if the convex hull of a finite set of points is compact. Here is a recent result, however that gives some good information. Let $B\left(X_{0}, R\right)$ denote the ball of radius $R$ centered at $X_{0}$ and let $S\left(X_{0}, R\right)$ be the sphere.

TheOREM 3.6. ([LR]) There is a constant $K$ such that for each $X_{0}, R$, the ball $B\left(X_{0}, R\right)$ is $K$-quasiconvex.

This means that for any pair of points $X, Y \in B\left(X_{0}, R\right)$, the geodesic joining $X, Y$ stays in the ball $B\left(X_{0}, R+K\right)$. The main step is to show that for each simple closed curve $\alpha$, the extremal length of $\alpha$ is a quasi-convex function along Teichmüller geodesics. This means that there is a number $K$ depending only on the topology of the surface, such that for each Teichmüller geodesic segment $r:[a, b] \rightarrow \operatorname{Teich}(S)$, each $a \leq t \leq b$, and any simple closed curve $\alpha$,

$$
\operatorname{Ext}_{r(t)}(\alpha) \leq K \max \left(\operatorname{Ext}_{r(a)}(\alpha), \operatorname{Ext}_{r(b)}(\alpha)\right)
$$

The proof is intricate because in general the flat length of a curve does not give a good estimate for the extremal length. Along a Teichmüller geodesic the flat length of a curve is a strictly convex function, while it is possible to find examples where the extremal length is essentially constant along long segments of a geodesic. The quasi-convexity of balls follows from the quasi-convexity of length functions and Kerckhoff's distance formula (1).

Another question that has been recently answered is the following. Given any pair of points $X, Y$ on the sphere $S\left(X_{0}, R\right)$ of radius $R$ centered at $X_{0}$, 
GEOMETRY OF TEICHMÜLLER SPACE WITH THE TEICHMÜLLER METRIC 303

we may ask for paths $\gamma(X, Y)$ joining $X, Y$ that stay outside $B\left(X_{0}, R\right)$. In a negatively curved space, this distance grows exponentially; that is, there exists $c_{1}>0, c_{2}>0$ such that for all $X_{0}, R$

$$
\sup _{X, Y \in S\left(X_{0}, R\right)} \inf _{\gamma(X, Y)}|\gamma(X, Y)| \geq c_{1} e^{c_{2} R} .
$$

However we have in $\operatorname{Teich}(S)$ the following result.

Theorem 3.7. ([DR]) For Teich $(S)$ with the Teichmüller metric, for any $X_{0}$ there are constants $c_{1}, c_{2}>0$ such that

$$
c_{1} R^{2} \leq \sup _{X, Y \in S\left(X_{0}, R\right)} \inf _{\gamma(X, Y)}|\gamma(X, Y)| \leq c_{2} R^{2} .
$$

That is, for any $X, Y \in S\left(X_{0}, R\right)$ there is a path joining $X, Y$ outside $S\left(X_{0}, R\right)$ of length at most $c_{2} R^{2}$, and there exists $X, Y$ such that the shortest path has length at least $c_{1} R^{2}$.

The proof uses the existence of chains of flats joining $X, Y$ and Minsky's product formula.

One can ask the question of when two geodesic rays $r_{1}, r_{2}$ stay bounded distance apart. This question has been answered completely. First we say that a measured foliation is minimal if all of its leaves are dense in one (or both) directions. We say it is uniquely ergodic if it is minimal and the transverse measure is unique up to scalar multiplication. If it is minimal and not uniquely ergodic then it is known that the set of transverse measures forms a finite dimensional convex set. The extreme points of the convext set are ergodic measures. Here is a result that combines results of [M1], [M2], $[\mathbf{I 2}],[\mathbf{L M}]$.

THEOREM 3.8. Suppose $r_{1}, r_{2}$ are rays determined by quadratic differentials $q_{1}, q_{2}$. Suppose they do not coincide on an infinite segment.

- If $q_{1}, q_{2}$ are both Strebel differentials and determine the same homotopy classes of cylinders then the rays stay bounded distance apart.

- If the vertical foliations $F_{v}\left(q_{1}\right)$ and $F_{v}\left(q_{2}\right)$ coincide up to scalar multiplication and are uniquely ergodic, then the rays stay bounded distance apart.

- Suppose $F_{v}\left(q_{1}\right)$ and $F_{v}\left(q_{2}\right)$ are minimal and topologically equivalent, but not uniquely ergodic. Then if the transverse measures are absolutely continuous with respect to each other, the rays stay bounded distance apart.

- In all other cases the rays diverge.

The hypothesis is equivalent to saying that $q_{2}$ is not the terminal quadratic differential for the Teichmüller geodesic ray determined by $q_{1}$ and vice versa. 


\section{Teichmüller geodesic flow}

We continue with a short discussion of properties of the Teichmüller geodesic flow. We present only a few of the many results in the subject that give a flavor of the subject. They fall into the theme of the negative curvature of Teichmüller space. Again let $Q_{\hat{k}}$ be a stratum. A stratum need not be connected although now the connected components are known $([\mathbf{K Z}]$, $[\mathbf{L a}],[\mathbf{B L}])$. We first have $([\mathbf{M} \mathbf{4}],[\mathbf{V}])$

THEOREM 4.1. $\mu\left(\mathbf{Q}_{\hat{k}}^{1}\right)<\infty$ and the action of the Teichmuller flow $g_{t}$ is ergodic on each component of each stratum.

There has been a great deal of beautiful work done in recent years. We do not list all of the results but just two of the most striking. The first is due to Avila and Viana. ([AV])

THEOREM 4.2. On every component of each stratum of Abelian differentials the nontrivial Lyapunov exponents of the flow are distinct.

We also have

TheOREM 4.3. ([AGY $])$ The Teichmüller flow $g_{t}$ is exponentially mixing on each stratum $\mathbf{Q}_{\hat{k}}=\mathbf{Q}\left(k_{1}, \ldots, k_{p},+\right)$ of Abelian differentials. There is $\delta>$ $0, C>0$ such that for compactly supported $C^{1}$ functions $U, V: \mathbf{Q}_{\hat{k}} \rightarrow \mathbf{R}$ we have,

$$
\begin{aligned}
& \left|\int_{Q_{\hat{k}}} U(q) V \circ g_{t}(q) d \mu_{\hat{k}}(q)-\int U(q) d \mu_{\hat{k}}(q) \int V(q) d \mu_{\hat{k}}(q)\right| \\
& \quad \leq C\|U\|_{C^{1}}\|V\|_{C_{1}} e^{-\delta t} .
\end{aligned}
$$

(The authors actually have a stronger statement that allows for functions not be compactly supported.)

\section{Counting problems in the mapping class group}

Now recall $\mu_{\hat{k}}$ the measure on each stratum $\mathrm{QD}_{\hat{k}}$. Assume $S$ is compact. We are interested in the principle stratum $k=(1, \ldots, 1,-)$ and denote the corresponding measure simply by $\mu$. let $\pi: \mathrm{QD}_{\hat{k}} \rightarrow \operatorname{Teich}(S)$ the natural projection which assigns to each quadratic differential the corresponding Riemann surface. Then $\nu=: \pi_{*}(\mu)$ defines a volume element on Teichmüller space.

TheOrEm 5.1. ([ABEM]) There is a function $\Lambda: \operatorname{Teich}(S) \rightarrow \mathbf{R}$ such that

$$
\nu\left(B(X, R) \sim \Lambda(X) e^{(6 g-6+2 n) R}\right)
$$

as $R \rightarrow \infty$. 
GEOMETRY OF TEICHMÜLLER SPACE WITH THE TEICHMÜLLER METRIC 305

(The symbol $\sim$ means that the ratio approaches 1 .)

The function $\Lambda$ can be described as follows. Let $\mathcal{M F}$ denote Thurston's space of measured foliations $([\mathbf{F L P}])$ and let $\mathcal{P} M F$ be the corresponding projective space of measured foliations, where we have identified two measured foliations if they differ by a scalar multiple. There is a natural Thurston measure $\mu^{T H}$ on $\mathcal{M F}$ invariant under the action of $\operatorname{Mod}(S)$. For each measured foliation $F$ and $X \in \operatorname{Teich}(S)$ there is a unique quadratic differential $q=q(F, X) \in \mathrm{QD}(X)$ such that $F_{v}(q)=F$. One can consider the set $B(X)$ of foliations $F \in \mathcal{M F}$ such that $\|q(F, X)\| \leq 1$. Then

$$
\Lambda(X):=\mu^{T H}(B(X)) .
$$

This theorem is part of a project to count lattice points in a ball. Fix points $X, Y \in \operatorname{Teich}(S)$ and set

$$
\operatorname{Mod}(X, Y, R)=\{f \in \operatorname{Mod}(S): f(Y) \in B(X, R)\} .
$$

Let $h=6 g-6+2 n$. (This is the topological entropy of the Teichmüller geodesic flow.)

Theorem 5.2. $([\mathbf{A B E M}])|\operatorname{Mod}(X, Y, R)| \sim \Lambda(X) \Lambda(Y) e^{h R}$ as $R \rightarrow \infty$.

A Pseudo-Anosov element $f \in \operatorname{Mod}(S)$ determines a pair of transverse foliations $F_{a}, F_{r}$ with the property that $f\left(F_{a}\right)=e^{2 \lambda} F_{a}$ and $f\left(F_{r}\right)=e^{-2 \lambda} F_{r}$ for some $\lambda>0$. By a theorem of Bers ([B3]) $f$ determines an axis in Teich $(S)$. This is a Teichmüller geodesic $A_{f}$ left invariant under the action of $f$ and such that $f$ translates points along $A_{f}$ by a Teichmüller distance equal to $\lambda$. In fact the geodesic is determined by a quadratic differential $q$ whose horizontal foliation is $F_{a}$ and whose vertical foliation is $F_{r}$. If $g$ is conjugate to $f$, then the conjugating element takes $A_{g}$ to $A_{f}$ and the translation lengths along these axes coincide. The conjugacy class $[f]$ then defines a closed geodesic in $\mathcal{M}(S)$ of length $\lambda$. One can then consider $\mathcal{P} A(\lambda)$ the set of conjugacy classes of Pseudo-Anosov elements $[f]$ whose corresponding closed geodesic has length at most $\lambda$. This is the same as the set of conjugacy classes of Pseudo-Anosovs whose expansion factor at most $e^{2 \lambda}$.

TheOREM 5.3. ([EM]) $|\mathcal{P} A(\lambda)| \sim \frac{e^{h \lambda}}{h \lambda}$ as $\lambda \rightarrow \infty$.

\section{Rays and the Thurston boundary}

We recall some of the basic fundamental theorems of Thurston as described in $[\mathbf{F L P}]$. Again denote $\mathcal{S}$ the homotopy classes of homotopically nontrivial simple closed curves with the discrete topology. We form the projective space $\mathcal{P} R^{\mathcal{S}}$ with the product topology. There is an embedding $\operatorname{Teich}(S) \rightarrow \mathcal{P} R^{\mathcal{S}}$ which sends $X \in \operatorname{Teich}(S)$ to the function

$$
\gamma \rightarrow l_{X}(\gamma)
$$


There is also an embedding of $\mathcal{P} M F$ into $\mathcal{P} R^{\mathcal{S}}$ which sends each projective class of measured foliation $[F]$ to the (projective class of ) function

$$
\gamma \rightarrow i(F, \gamma)
$$

where $i(\cdot, \cdot)$ is the geometric intersection number of measured foliations with homotopy classes of simple closed curves.

Thurston proved that with these embeddings $\mathcal{P} M F$ is the boundary of $\operatorname{Teich}(S)$ and the union is a closed ball. We can ask how the Teichmüller geometry fits in with the Thurston compactification. The issue here is that Teichmüller rays are defined in complex analytic terms by quadratic differentials, while the Thurston embedding is via hyperbolic geometry. Again there are some older results and new results.

THEOREM 6.1. ([M3]) Suppose $r(t)$ is a ray defined by the quadratic differential $q$.

- If $F_{v}(q)$ is uniquely ergodic then $r(t)$ converges to the same projective class $\left[F_{v}(q)\right] \in \mathcal{P} M F$ as $t \rightarrow \infty$.

- If $q$ is a Strebel quadratic differential such that $F_{v}(q)$ has cylinders in the homotopy classes of $\beta_{1}, \ldots, \beta_{p}$ then $r(t)$ converges to the (projective) measured foliation $[F]$ defined as follows. The leaves of $F$ are closed and are also in the homotopy classes of $\beta_{1}, \ldots, \beta_{p}$ and the heights of the cylinders are all equal.

The second statement means that if $\alpha$ is any simple closed curve then $i(F, \alpha)=c \sum i\left(\alpha, \beta_{i}\right)$ for some $c>0$.

TheOREM 6.2. $([\mathbf{L}])$ There are examples of rays such that $r(t)$ does not have a limit in $\mathcal{P} M F$

These rays can be described as follows. Choose two standard square flat tori $T_{1}, T_{2}$ of area 1 and segments $\lambda_{1}, \lambda_{2}$ of equal length on each. Let $s_{1}, s_{2}$ be the slopes of the segments. Assume $s_{1}$ has unbounded partial quotients in its continued fraction expansion and $s_{2}$ has bounded partial quotients. Let $F_{i}$ be the foliation on each $T_{i}$ by lines with slope $s_{i}$. Now rotate each torus so that $\lambda_{1}, \lambda_{2}$ are vertical and the $F_{i}$ are now in the vertical direction on the rotated tori. Slit each torus along $\lambda_{i}$ and glue pairwise. The resulting quadratic differential has genus 2 and has double zeroes at the endpoints of the glued $\lambda_{i}$. The union of the glued $\lambda_{i}$ are a pair of saddle connections that separate the surface into $T_{1}$ and $T_{2}$. The vertical trajectories on each torus are dense on that torus. Along the corresponding ray $r(t)$ the separating curve $\gamma$ is pinched. Each representative $F$ of any limiting foliation in $\mathcal{P} M F$ has the following properties. It satisfies $i(F, \gamma)=0$. Moreover $F$ restricted to $T_{i}$ is a multiple $r_{i} F_{i}$ of $F_{i}$. However what is proved is that there are limiting $F$ and $F^{\prime}$ such that the corresponding ratios $\frac{r_{2}}{r_{1}} \neq \frac{r_{2}^{\prime}}{r_{1}^{\prime}}$. This means that the projective classes of $F$ and $F^{\prime}$ are different. 


\section{Combinatorial Description of the Teichmüller metric}

In this section we describe some work of K. Rafi ([Ra]) on the Teichmüller metric. He gives a formula for the distance between two points in terms of combinatorial data of subsurface projections. This work can be thought of as a higher genus analogue of Artin's work that gives the itinerary of a geodesic on the modular curve in terms of the continued fraction expansion of the endpoint on the real line of the geodesic. To describe subsurface projections, let $Y \subseteq S$ be an essential subsurface. Previously we described the curve complex $\mathbf{C}(S)$ of a surface. For a subsurface $Y$ we have the arc and curve complex $\mathbf{C}(Y)$ of $Y$. The vertices of $\mathbf{C}(Y)$ are isotopy classes of essential arcs and simple closed curves contained in $Y$. An essential arc has its endpoints on $\partial Y$. If $Y$ is not an annulus, the isotopy is rel $\partial Y$. If $Y$ is an annulus then isotopy means rel endpoints. Two vertices of $\mathbf{C}(Y)$ are joined by an edge if they have disjoint representatives.

Let $f: S_{Y} \rightarrow S$ be the regular cover such that $f_{*}\left(\pi_{1}\left(S_{Y}\right)\right)$ is conjugate to $\pi_{1}(Y)$. For any collection of curves $\nu \subset \mathbf{C}(S)$, let $\tilde{\nu}$ be their lift to $S_{Y}$. The components of $\tilde{\nu}$, if any, that are essential arcs in $S_{Y}$ or closed curves in $S_{Y}$ form a subset of $\mathbf{C}\left(S_{Y}\right)$. We call this set $\nu_{Y}$, the projection of $\nu$ to $Y$. The surface $S_{Y}$ is homeomorphic to $Y$ and so we have a corresponding subset of $\mathbf{C}(Y)$. For $\nu, \nu^{\prime}$ any pair of subsets of $\mathbf{C}(S)$ we denote by $d_{Y}\left(\nu, \nu^{\prime}\right)$ the diameter in $\mathbf{C}(Y)$ of $\nu_{Y} \cup \nu_{Y}^{\prime}$. If $Y$ is an annulus in the class of the curve $\alpha$, we write $d_{\alpha}$ for this diameter.

A marking on a surface consists of a collection of $6 g-6+2 n$ simple closed curves $\alpha_{1}, \ldots, \alpha_{3 g-3+n}, \beta_{1}, \ldots, \beta_{3 g-3}$. The $\alpha_{i}$ are pairwise disjoint and are called the base of the marking. Each transversal curve $\beta_{i}$ is disjoint from $\alpha_{j}, j \neq i$ and intersects $\alpha_{i}$ minimally. Now for any $X \in \operatorname{Teich}(S)$, given its hyperbolic metric, we associate a short marking $\mu(X)$ to $X$ as follows. We choose the shortest curve $\alpha_{1}$ on the surface and then the shortest curve $\alpha_{2}$ disjoint from $\alpha_{1}$ and so forth until we have a base for the marking. Then for each $\alpha_{j}$, we let $\beta_{j}$ be the shortest transversal. There are a bounded number of shortest markings for $\sigma$.

Definition 7.1. Fix $\epsilon>0$ small. We say $X \in \operatorname{Teich}(S)$ belongs to the $\epsilon$-thick part of $\operatorname{Teich}(S)$ if it does not belong to $\Omega_{\mathcal{C}}(\epsilon)$ for any collection of simple closed curves $\mathcal{C}$. (Recall this means that the shortest curve on $X$ has hyperbolic length at least $\epsilon)$.

Given $k>0$ denote by $[x]_{k}$ the function which is equal to $x$ if $x \geq k$ and 0 otherwise. Also adopt the notation that for two quantities, $x \asymp y$, if there are constants $C_{1}, C_{2}>0$ just depending on $(g, n)$ such that

$$
\frac{1}{C_{1}} x-C_{2} \leq y \leq C_{1} x+C_{2}
$$


TheOREM 7.2. There is $k>0$ such that given $X_{1}, X_{2}$ in the $\epsilon$-thick part of $\operatorname{Teich}(S)$,

$$
d_{T}\left(X_{1}, X_{2}\right) \asymp \sum_{Y \subseteq S}\left[d_{Y}\left(\mu\left(X_{1}\right), \mu\left(X_{2}\right)\right)\right]_{k}+\sum_{\alpha} \log \left[d_{\alpha}\left(\mu\left(X_{1}\right), \mu\left(X_{2}\right)\right)\right]_{k} .
$$

The first sum is over subsurfaces $Y$ that are not annuli and the second sum is over annuli.

If either $X_{1}$ or $X_{2}$ is not in the $\epsilon$-thick part we have to add terms as follows. If there is a collection of curves $\mathbf{C}$ such that both points are in $\Omega_{\mathbf{C}}(\epsilon)$ then there is a term as in Minsky's formula for how far apart they are in $\Omega_{\mathbf{C}}(\epsilon)$. For each curve that is short on one surface and on not the other there is a term corresponding to the distance to a surface for which that curve has length $\epsilon$. We refer to Theorem 6.1 of [Ra] for details.

One can say more about the itinerary of geodesics in terms of the subsurface projections. Again let $r:[a, b] \rightarrow \operatorname{Teich}(S)$ the Teichmüller geodesic joining $X_{1}$ and $X_{2}$ parametrized by arclength.

Theorem 7.3. ([Ra]) There are constants $M_{1}, M_{2}, 0<\epsilon_{1}<\epsilon_{0}$ depending only on topology, such that for each subsurface $Y$, if $d_{Y}(r(a), r(b)) \geq M_{1}$ there is a nonempty connected interval $I_{Y}$ such that

- For $[c, d] \cap I_{Y}=\emptyset d_{Y}\left(\mu(r(c), \mu(r(d))) \leq M_{2}\right.$.

- For any $t \in I_{Y}$ the hyperbolic length of $\partial Y$ on $r(t)$ is at most $\epsilon_{0}$.

- For $t \notin I_{Y}$ the hyperbolic length of $\partial Y$ on $r(t)$ is at least $\epsilon_{1}$.

The first conclusion says that up to a constant one only changes projection to $Y$ while in $I_{Y}$. The second conclusion says that while in $I_{Y}$ the curves in $\partial Y$ are short and the third says that lengths are bounded below outside $I_{Y}$.

\section{Geometry of moduli space and the Deligne-Mumford compactification}

In this section I will describe some recent work joint with Benson Farb. $([\mathbf{F M}])$ The work here was inspired by the work in $[\mathbf{J M}]$. The Teichmüller metric descends to a complete metric $d_{\mathcal{M}(S)}$ on the quotient moduli space.

Deligne-Mumford [DM] constructed a compactification $\overline{\mathcal{M}(\mathrm{S})}^{\mathrm{DM}}$ of $\mathcal{M}(S)$ whose points are represented by conformal structures on noded Riemann surfaces. They proved that $\overline{\mathcal{M}(\mathrm{S})}^{\mathrm{DM}}$ is a projective variety. As such, $\overline{\mathcal{M}(\mathrm{S})}^{\mathrm{DM}}$ as a topological space comes with a natural stratification: each stratum is a product of moduli spaces of surfaces of lower complexity. We will equip each moduli space with the Teichmüller metric, and the product of moduli spaces with the sup metric. In this way $\overline{\mathcal{M}(\mathrm{S})}^{\mathrm{DM}}$ has the structure of a metric stratified space, i.e., a stratified space with a metric on each stratum. We note that $\overline{\mathcal{M}(\mathrm{S})}^{\mathrm{DM}}$ was also constructed topologically by Bers in [B2]. 
We reconstruct the Deligne-Mumford compactification (as a metric stratified space) purely from the intrinsic metric geometry of $\mathcal{M}(S)$ endowed with the Teichmüller metric. We then formalize this procedure by creating a "functor" for a quite general class of geodesic metric spaces, which associates to the space a certain iterated ray space. When applied to moduli space this gives the Deligne Mumford compactification. To begin, we again consider Strebel rays.

Again let $q$ be a Strebel differential on a surface of genus $g$ with $n$ punctures. Associated to a Strebel ray $r$ is an endpoint $r(\infty)$ which lives in a lower dimensional moduli space. The point $r(\infty)$ is constructed as follows. Let $C_{1}, \ldots, C_{p}$ be the cylinders defined by $q$. Cut each $C_{i}$ along one of the closed vertical trajectores. Glue into each side of the cut a half infinite cylinder. The result is a (possibly disconnected) surface $r(\infty)$ with a total of $2 p+n$ punctures.

We are particularly interested in Strebel rays such that the quadratic differential has a single cylinder. In that case $r(\infty)$ is connected if the homotopy class of the closed curve $\alpha$ is a nonseparating curve, and $r(\infty)$ has two components if it is separating. Let $\mathcal{M}(S \backslash \alpha)$ be the moduli space of the corresponding surface. If $S \backslash \alpha$ is disconnected, then $\mathcal{M}(S \backslash \alpha)$ is a product of two moduli spaces and we give it the sup metric of the corresponding Teichm'uller metrics. There are a finite number of possible boundary moduli spaces determined by the topological type of the curve $\alpha$.

TheOREM 8.1. Suppose $r_{1}, r_{2}$ are Strebel rays defined by one cylinder Strebel differentials. If $r_{1}(\infty)$ and $r_{2}(\infty)$ belong to the same boundary moduli space then there are initial points $r_{1}(0), r_{2}(0)$ such that $\lim _{t \rightarrow \infty} d\left(r_{1}(t), r_{2}(t)\right)$ exists and is a minimum among all choices of basepoints. Furthermore the limit coincides with the Teichmüller distance between $r_{1}(\infty)$ and $r_{2}(\infty)$ in the boundary moduli space $\mathcal{M}(S \backslash \alpha)$.

We can define a distance function $d(\cdot, \cdot)$ defined on asymptote classes of one cylinder Strebel rays $[r]$ by saying the distance between asymptote classes is given by the above limit defined by any representatives of the classes. This space has several components; one component corresponding to rays defined by Strebel differentials where the cylinder is a nonseparating curve and one component for each homeomorphism type of separating curve. By the above Theorem then this space is naturally isometric to the union of the moduli spaces $\mathcal{M}(S \backslash \alpha)$ where $\alpha$ is allowed to run over all homeomorphism classes of curves.

We may now iterate this procedure by defining asymptote class of one cylinder Strebel rays on $\cup_{\alpha} \mathcal{M}(S \backslash \alpha)$. The next lower dimensional union of moduli spaces is then the space of one cylinder Strebel rays on $\cup_{\alpha} \mathcal{M}(S \backslash \alpha)$. This can be thought of as a space of "Strebel rays on rays" on $\mathcal{M}(S)$. Continuing, then we can construct the compactification as an iterated space of rays on rays. 
We now introduce a functor for any geodesic metric space $X$ that satisfies several assumptions. This will be a space $\bar{X}^{i r}$ of $X$, called the iterated EDM ray space associated to $X$. The space $\bar{X}^{i r}$ will be constructed via equivalence classes of what are called isolated EDM rays, and will have the structure of a metric stratified space. The above results prove that this functor applied to $\mathcal{M}(S)$ produces the Deligne-Mumford compactification $\overline{\mathcal{M}(\mathrm{S})}^{\mathrm{DM}}$; that is, there is a stratification-preserving homeomorphism from $\overline{\mathcal{M}}(S)^{i r}$ to the Delgine-Mumford compactification $\overline{\mathcal{M}(\mathrm{S})}^{\mathrm{DM}}$ which is an isometry on each stratum.

We are given a metric space $(X, d)$. By a geodesic we mean a locally distance minimizing image of a finite or infinite segment in $\mathbf{R}$.

Definition 8.2 (EDM rays). A ray $r:[0, \infty) \rightarrow X$ in a metric space $X$ is eventually distance minimizing, or EDM, if there exists $t_{0}$ such that for all $t \geq t_{0}$ :

$$
d\left(r(t), r\left(t_{0}\right)\right)=\left|t-t_{0}\right|
$$

Note that, if $r$ is an EDM ray, after cutting off an initial segment of $r$ we obtain a globally geodesic ray, i.e., an isometric embedding of $[0, \infty) \rightarrow X$.

TheOrem 8.3. A ray $r$ in $\mathcal{M}(S)$ is an EDM ray iff it is a Strebel ray.

There is another definition of independent interest which will not however be used in the definiton of the iterated ray space.

Definition 8.4 (ADM rays). The ray $r(t)$ is almost distance minimizing, or $\mathrm{ADM}$, if there are constants $C, t_{0} \geq 0$ such that for $t \geq t_{0}$ :

$$
d\left(r(t), r\left(t_{0}\right)\right) \geq\left|t-t_{0}\right|-C
$$

It is easy to check that a ray $r$ is ADM if and only if, for every $\epsilon>0$ there exists $t_{0} \geq 0$ so that for all $t \geq t_{0}$ :

$$
d\left(r(t), r\left(t_{0}\right)\right) \geq\left|t-t_{0}\right|-\epsilon
$$

THEOREM 8.5. Let $r$ be a ray in $\mathcal{M}(S)$. Then $r$ is ADM if and only if it is mixed Strebel ray.

Recall in the previous discussion we considered one cylinder Strebel rays. We make a general definition which in the case of $\mathcal{M}(S)$ will give exactly these rays. Suppose now $(X, d)$ can be written as $X_{1} \times \ldots X_{m}$ of metric spaces $\left(X_{i}, d_{i}\right)$ and the metric $d$ is the sup metric. We will consider rays in each factor. We say that a ray $r$ is isolated if the following two properties hold

(1) there is a factor $X_{j}$ such that $r \subset X_{j}$ ( $r$ is constant on other factors) and $r$ is an EDM ray in $X_{j}$. 
GEOMETRY OF TEICHMÜLLER SPACE WITH THE TEICHMÜLLER METRIC 311

(2) for every $p \in X_{j}$, the set of asymptote classes of EDM rays $\left[r^{\prime}\right] \subset$ $X_{j}$ which are a bounded distance from $r$, and which have some representative passing through $p$, is countable.

Proposition 8.6. A ray in $\mathcal{M}(S)$ is an isolated EDM ray if and only if it is a one-cylinder Strebel ray.

Now consider the set $\operatorname{Asy}(X)$ of all asymptote classes of isolated EDM rays $[r]$ in $X$. We assume that for any two such rays $r_{1}, r_{2}$ there is a choice of basepoint on each such that $\lim _{t \rightarrow \infty} d\left(r_{1}(t), r_{2}(t)\right)$ exists. With that assumption we can endow $\operatorname{Asy}(X)$ with a distance function via

$$
d_{\text {asy }}\left(\left[r_{1}\right],\left[r_{2}\right]\right)=\lim _{t \rightarrow \infty} d\left(r_{1}(t), r_{2}(t)\right)
$$

for a choice of basepoints that minimizes this limit. It is easy to check that in general this defines a metric.

We also assume that if $A s y(X)$ can be written as a product of factors, then up to a permutation, it is written uniquely as a product. It probably holds under quite general conditions on $(X, d)$. Now we wish to define the iterated ray space.

Let $\left(D_{0}(X), d_{0}\right):=(X, d)$.

Step 1 (Inductive step): Suppose we are given the metric space $D_{k}(X)$, written as a product of factors $X_{1} \times \ldots \times X_{m}$ with the metric $d_{k}(\cdot, \cdot)$, where $d_{k}$ is the sup of the metrics $d^{j}$ of the factors. If none of the factors $X_{j}$ contains isolated EDM rays, define $D_{m}(X)=\emptyset$ for all $m>k$ and stop the inductive process. If some factor $X_{j}$ contains isolated rays then we set

$$
D_{k+1}^{j}(X)=X_{1} \times \ldots \times X_{j-1} \times \operatorname{Asy}\left(X_{j}\right) \times X_{j+1} \times \ldots \times X_{m} .
$$

We can endow $D_{k+1}^{j}(X)$ with a distance function $d_{k+1}^{j}$ as the sup metric on the factors. From the above assumption, we note that if a component of $\operatorname{Asy}\left(X_{j}\right)$ is a product, then it can be written uniquely as a product. Thus, given the product representation of $D_{k}(X)$, we have a unique product representation of $D_{k+1}^{j}(X)$.

Note also that if two points in $D_{k+1}^{j}(X)$ have an infinite distance from each other, then they are in different components of $D_{k+1}^{j}(X)$. We then set

$$
D_{k+1}(X)=\sqcup_{j=1}^{m} D_{k+1}^{j}(X)
$$

with metric $d_{k+1}$ which is the corresponding metric $d_{k+1}^{j}$ on each term in the disjoint union.

Iterating this procedure the result is a space $\bar{X}^{i r}$ which then has the structure of a metric stratified space. Our results show

THEOREM 8.7. Applied to $\mathcal{M}(S)$ there is a strata-preserving homeomorphism $\overline{\mathcal{M}(S)}^{i r} \rightarrow \overline{\mathcal{M}(\mathrm{S})}{ }^{\mathrm{DM}}$ which is an isometry on each stratum. 


\section{References}

[A] L.V. Ahlfors, Lectures on quasi-conformal mappings, University Lecture Series, American Mathematical Society, v. 38 (2006).

[ABEM] J. Athreya, A. Bufetov, A. Eskin, M. Mirzakhani, Lattice Point Asymptotics and Volume Growth on Teichmuller space, arXiv:math/0610715.

[AGY] A. Avila, S. Gouezel, J-C Yoccoz, Exponential mixing for the Teichmuller flow arXiv:math/0511614.

[AV] A. Avila, M. Viana, Simplicity of Lyapunov spectra: proof of the ZorichKontsevich conjecture arXiv:math/0508508.

[B1] L. Bers, Quasiconformal mappings and Teichmüller's theorem, in Analytic Functions, (R. Nevanlinna et al eds.) Princeton University Press (1960).

[B2] L. Bers, Spaces of degenerating Riemann surfaces, in Discontinuous groups and Riemann surfaces (Proc. Conf., Univ. Maryland, College Park, Md., 1973), pp. 43-55. Ann. of Math. Studies, No. 79, Princeton Univ. Press, Princeton, N.J., 1974.

[B3] L. Bers, An extremal problem for quasiconformal mappings and a theorem by Thurston, Acta Math. 141, (1978) 73-98.

[BL] C. Boissy, E. Lanneau, Dynamics and geometry of the Rauzy-Veech induction for quadratic differentials,arXiv:0710.5614.

[DM] P. Deligne, D. Mumford, The irreducibility of the space of curves of given genus, Publ. Math. IHES, 36 (1969), 75-109.

[DR] M. Duchin, K. Rafi, Divergence of Geodesics in Teichmuller Space and the Mapping Class Group . arXiv:math/0611359.

[EM] A. Eskin, M. Mirzakhani, Counting closed geodesics in Moduli space, arXiv:0811.2362.

[FMa] B. Farb, D. Margalit, A primer on mapping class groups.

[FM] B. Farb, H. Masur, Teichmüller geometry of moduli space, distance minimizing rays and the Deligne-Mumford compactification, arXiv:0803.0141.

[FLP] A. Fathi, F. Laudenback, V. Poenaru, Travaux de Thurston sur les surfaces, Astérisque 66-67, (1979).

[G] F.P. Gardiner, Teichm"uller theory and quadratic differentials Wiley-Interscience (1987).

$[\mathrm{H}] \quad$ J. Hubbard, Teichmüller theory and applications to geometry, topology, and dynamics I, Matrix Editions.

[I1] N. Ivanov, Subgroups of Teichmüller modular groups Translations of Mathematics, Monographs, v. 115 American Mathematical Society (1992).

[I2] N. Ivanov Isometries of Teichmller spaces from the point of view of Mostow rigidity, Topology, ergodic theory, real algebraic geometry, ed. by V. Turaev and A. Vershik, Amer. Math. Soc. Transl. Ser. 2, V. 202, American Mathematical Society, 2001, 131-149.

[JM] L. Ji, R. MacPherson, Geometry of compactifications of locally symmetric spaces, Ann. Inst. Fourier, Grenoble, . 52, No. 2 (2002), 457-559.

[Ke] S. Kerckhoff, The asymptotic geometry of Teichmüller space, Topology 19 (1981) 23-41.

[KZ] M. Kontsevich, A. Zorich, Connected components of spaces of Abelian differentials with prescribed singularities Inventiones Math 153 631-683 (2003).

[La] E. Lanneau Connected components of the strata of the moduli spaces of quadratic differentials arXiv:math/0506136.

[L] A. Lenzhen, Teichmuller geodesics that don't have a limit in PMF, arXiv:math/0511001.

[LM] A. Lenzhen, H. Masur, Divergence of Teichmueller Geodesics, arXiv:0803.1867.

[LR] A. Lenzhen, K. Rafi, in preparation. 
GEOMETRY OF TEICHMÜLLER SPACE WITH THE TEICHMÜLLER METRIC 313

[M1] H. Masur, On a Class of Geodesics in Teichmüller space, Annals of Math 102 (1975) 205-221.

[M2] H. Masur, Uniquely ergodic quadratic differentials, Comment Math. Helvetici, 55 (1980), 255-266.

[M3] H. Masur, Uniquely ergodic quadratic differentials, Comment Math. Helvetici 55 (1980) 255-266.

[M4] H. Masur, Interval exchange transformations and measured foliations Annals of math. 115 169-200 (1982).

[MM] H. Masur, Y. Minsky, Geometry of the Complex of Curves I: Hyperbolicity, Invent.Math 138 (1999) 103-149.

[MW] H. Masur, M. Wolf, Teichmuller Space is Not Gromov Hyperbolic.

[Mi] Y. Minsky, Extremal length estimates and product regions in Teichmüller space, Duke Math Journal 83 (1996), 249-286.

[P] A. Papadopoulos Ed. Handbook of Teichmüller theory I European Mathematical Society (EMS), Zurich, (2007).

[Ra] K. Rafi, A combinatorial model for the Teichmüller metric, GAFA 17 (2007) 936-959.

[St] K. Strebel, Quadratic differentials Ergebnisse der Math 5, Springer-Verlag (1984).

[V] W. Veech, Gauss measures for transformations on the space of interval exchange transformations, Annals of Math 115 215-242 (1982).

[W] S. Wolpert, The Weil-Petersson metric geometry arXiv:0801.0175.

Dept. of Mathematics, University of Chicago, Chicago, IL 60637

E-mail address: masur@math.uchicago.edu 
